\title{
Utilizzo della tensione di picco per la verifica a fatica dei giunti saldati d'angolo con il metodo degli elementi finiti
}

\author{
G. Meneghetti \\ Università di Padova, Dipartimento di Ingegneria Meccanica, giovanni.meneghetti@unipd.it
}

Riassunto. In questo lavoro viene applicato il metodo della tensione di picco per l'analisi della resistenza a fatica di giunti saldati con cordone d'angolo limitatamente al caso di rottura al piede del cordone di saldatura. Il metodo è un'applicazione ingegneristica dell'approccio locale basato sul fattore di intensificazione delle tensioni per intagli (Notch-Stress Intensity Factor, N-SIF) di modo I, che assimila il profilo del piede del cordone di saldatura ad un intaglio a $\mathrm{V}$ con raggio di raccordo pari a zero. Inoltre si basa sull'utilizzo della tensione di picco singolare calcolata al piede del cordone mediante un'analisi agli elementi finiti lineare elastica con elementi aventi una prefissata dimensione, assunta pari a $1 \mathrm{~mm}$ in questo lavoro. La relativa semplicità di utilizzo e la robustezza del metodo lo rendono adatto all'applicazione in ambito industriale.

ABSTRACT. The paper presents the so-called Peak Stress Method applied to the analysis of the fatigue strength of fillet-welded joints failing from the weld toe. Such method is an engineering application of the local approach based on the mode I Notch Stress Intensity Factor (N-SIF), which assumes the weld toe profile as a sharp Vshaped notch having a toe radius equal to zero. According to the Peak Stress Method, the design stress is the elastic peak stress calculated at the point of singularity by means of a finite element analysis, where the element size has a fixed value, for example equal to $1 \mathrm{~mm}$ in the present work. Due to its simplicity, the Peak Stress Method proved to be convenient in practical applications.

KEYwORDs. Fatigue Design, Local Approaches, Notch-Stress Intensity Factors, Fillet Welded Joints, Finite Element Analysis, Coarse Mesh, Peak Stress Method.

\section{INTRODUZIONE}

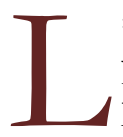

'approccio locale basato sui Fattori di Intensificazione delle Tensioni per intagli (Notch-Stress Intensity Factors, $\mathrm{N}-\mathrm{SIFs}$ ) è stato applicato con successo all'analisi della resistenza a fatica di giunti saldati, sia in acciaio che in lega leggera, con rottura al piede del cordone di saldatura [1-3]. L'assunzione di base è che il profilo geometrico del piede del cordone di saldatura si possa assimilare ad un intaglio acuto a $\mathrm{V}$ con angolo di apertura che nella maggior parte dei casi si può ritenere prossimo a $135^{\circ}$, come rappresentato in Figura 1. Questa assunzione è ragionevole in quanto il valore minimo del raggio di raccordo in giunti saldati d'angolo e testa-testa con tecnologie tradizionali varia notevolmente anche lungo uno stesso cordone di saldatura e i valori sono compresi tipicamente tra $0.05 \mathrm{~mm}$ e $0.6 \mathrm{~mm}$ [4]. La variabilità dei valori medi del raggio di raccordo è ancora maggiore e pertanto sarebbe poco rappresentativa la definizione di un valore caratteristico del raggio di raccordo per effettuare un'analisi a fatica.

Assunta la geometria di Figura 1, la distribuzione locale delle tensioni in un'analisi piana lineare elastica è descritta dagli NSIFs di modo I e II, $\mathrm{K}_{\mathrm{I}}^{\mathrm{V}}$ and $\mathrm{K}_{\mathrm{II}}^{\mathrm{V}}$, rispettivamente, che quantificano l'intensità della distribuzione asintotica delle tensioni in accordo alla soluzione teorica di Williams [5]. La definizione degli N-SIFs di modo I e II [6] è la seguente:

$$
\begin{aligned}
& \mathrm{K}_{\mathrm{I}}^{\mathrm{V}}=\sqrt{2 \cdot \pi} \cdot \lim _{\mathrm{r} \rightarrow 0}\left[\left(\sigma_{\theta \theta}\right)_{\theta=0} \cdot \mathrm{r}^{1-\lambda_{1}}\right] \\
& \mathrm{K}_{\mathrm{II}}^{\mathrm{V}}=\sqrt{2 \cdot \pi} \cdot \lim _{\mathrm{r} \rightarrow 0}\left[\left(\tau_{\mathrm{r} \theta}\right)_{\theta=0} \cdot \mathrm{r}^{1-\lambda_{2}}\right]
\end{aligned}
$$




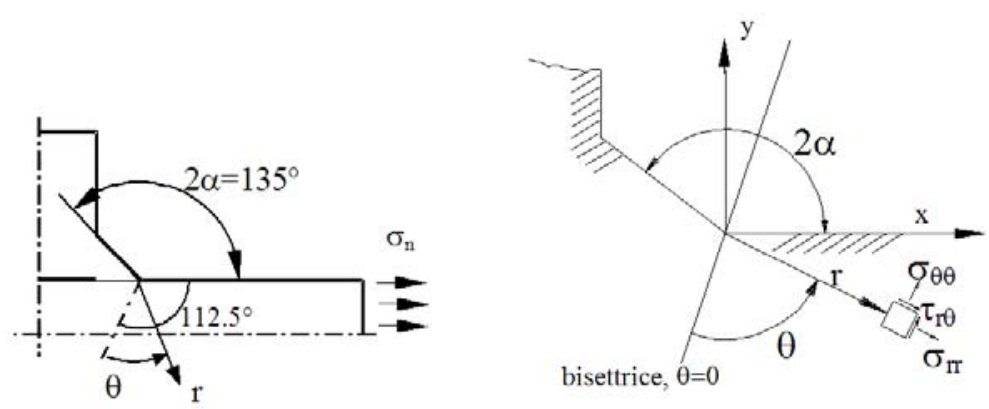

Figura 1: Modello geometrico di un giunto saldato d'angolo e sistema di riferimento polare con origine al piede del cordone.

Nelle precedenti definizioni, r e $\theta$ sono le coordinate polari mostrate in Fig. $1, \lambda_{1}, \lambda_{2}$ sono gli autovalori di Williams per modo I e II, rispettivamente. Quando l'angolo di apertura è pari a $135^{\circ}$, l'esponente $\left(1-\lambda_{1}\right)$ risulta 0.326 mentre $\left(1-\lambda_{2}\right)$ risulta -0.302 . Le componenti dello stato di tensione si possono infine esprimere nella forma [1]:

$$
\left\{\begin{array}{c}
\sigma_{\vartheta \vartheta} \\
\sigma_{\mathrm{rr}} \\
\tau_{\mathrm{r} \vartheta}
\end{array}\right\}=\mathrm{r}^{-0.326} \cdot \mathrm{K}_{\mathrm{I}}^{\mathrm{V}} \cdot\left\{\begin{array}{c}
\mathrm{f}_{1, \vartheta}(\vartheta) \\
\mathrm{f}_{1, \mathrm{r}}(\vartheta) \\
\mathrm{f}_{1, \mathrm{r} \vartheta}(\vartheta)
\end{array}\right\}+\mathrm{r}^{0.302} \cdot \mathrm{K}_{\mathrm{II}}^{\mathrm{V}} \cdot\left\{\begin{array}{c}
\mathrm{f}_{2, \vartheta}(\vartheta) \\
\mathrm{f}_{2, \mathrm{r}}(\vartheta) \\
\mathrm{f}_{2, \mathrm{r} \vartheta}(\vartheta)
\end{array}\right\}
$$

dove le funzioni f sono espressioni trigonometriche note. Le espressioni (2) mostrano che, essendo $2 \alpha=135^{\circ}$, solamente la distribuzione di modo I è singolare.

La Fig. 2 mostra come esempio il campo di tensione locale lungo la lamiera principale di un giunto saldato d'angolo con doppio irrigidimento. È evidente che solo il contributo di modo I allo stato di tensione locale è singolare, mentre il contributo di modo II tende a zero. Nella figura è anche riportato per confronto il risultato ottenuto con un'analisi piana lineare elastica agli elementi finiti. Utilizzando i risultati dell'analisi agli elementi finiti ottenuti lungo la bisettrice dell'intaglio e applicando la definizione (1a) è possibile calcolare $\mathrm{K}_{\mathrm{I}}^{\mathrm{V}}$, parametro da cui dipende la resistenza a fatica. Infatti esprimendo in sintesi l'approccio locale, è possibile affermare che due giunti saldati che in una prova di fatica sperimentano lo stesso range di variazione di $\mathrm{K}_{\mathrm{I}}^{\mathrm{V}}$ avranno la stessa vita a fatica, qualunque sia la loro geometria e la loro dimensione assoluta.

Tuttavia la Fig. 2 mostra che la densità della mesh necessaria per calcolare la distribuzione asintotica del campo locale, e quindi il fattore di intensificazione di modo I, è elevata. Pertanto le fasi di preparazione della mesh, di calcolo e di analisi dei risultati risultano dispendiose in termini di tempo. Si nota che in Fig. 2 la dimensione degli elementi più piccoli utilizzati è una frazione di micron. L'analisi agli elementi finiti risulta inoltre ancora più complicata per giunti aventi geometria tridimensionale come ad esempio i giunti con attacchi longitudinali.

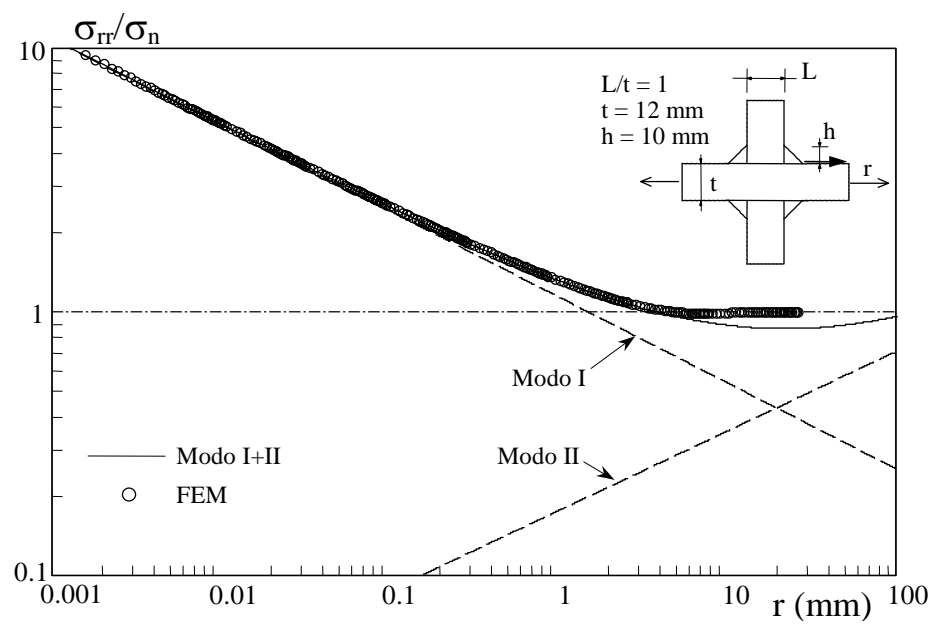

Figura 2: confronto fra risultati analitici e numerici nella valutazione dello stato tensionale lungo la superficie di un giunto saldato. 
Il metodo della tensione di picco supera questi problemi di applicazione dell'approccio N-SIF nell'analisi della resistenza a fatica dei giunti saldati, sostituendo al parametro $\mathrm{K}_{\mathrm{I}}^{\mathrm{V}}$ la tensione di picco singolare calcolata a piede cordone, che risulta più facile da calcolare con un'analisi agli elementi finiti. Pertanto dopo aver presentato le basi teoriche del metodo, ne verrà mostrata l'applicazione all'analisi della resistenza a fatica di giunti d'angolo aventi geometria semplice. Questa analisi consente di tarare due curve di resistenza a fatica in termini di tensione di picco, valide per giunti saldati d'angolo in acciaio e in lega leggera, rispettivamente, senza trattamenti post-saldatura. Successivamente queste curve di resistenza a fatica di progetto verranno utilizzate per stimare la resistenza a fatica di giunti aventi geometria più complessa.

\section{IL METODO DELLA TENSIONE DI PICCO}

$\mathrm{Q}$

uando si considera un componente contenente punti di singolarità, ad esempio l'apice di una cricca o di un intaglio aperto a spigolo vivo, la tensione calcolata all'apice con un'analisi lineare elastica agli elementi finiti dipende dalla fittezza della mesh, in modo che la tensione è tanto più elevata quanto minore è la dimensione degli elementi utilizzati. Pertanto la tensione singolare calcolata con gli elementi finiti non ha significato.

Tuttavia Nisitani e Teranishi [7] hanno mostrato che la tensione singolare $\sigma_{\text {peak }}$ calcolata all'apice di una cricca con un'analisi lineare elastica agli elementi finiti è in rapporto costante con il fattore di intensificazione delle tensioni $\mathrm{K}_{\mathrm{I}}$ $\left(\mathrm{K}_{\mathrm{I}} / \sigma_{\text {peak }}=\right.$ costante), purché sia mantenuta costante la dimensione e il tipo di elemento utilizzato.

Recentemente, è stata data una giustificazione teorica al metodo della tensione di picco (PSM) [8] ed il suo utilizzo è stato esteso a casi diversi da quelli considerati in origine da Nisitani e Teranishi. In particolare il PSM è stato esteso a casi di intagli aperti a $\mathrm{V}$ a spigolo vivo ed è stato valutato l'effetto della dimensione degli elementi utilizzati per l'analisi. La Fig. 3 mostra diverse geometrie contenenti punti di singolarità dovuti a cricche o intagli a spigolo vivo. Per ognuna di queste geometrie è stata fatta variare la profondità dell'intaglio o della cricca a parità di dimensione di elemento oppure la dimensione dell'elemento a parità di dimensione di intaglio o cricca. Le analisi agli elementi finiti lineari elastiche sono state fatte con il codice Ansys ${ }^{\circledR}$ utilizzando gli elementi quadrangolari a quattro nodi PLANE 42. La mesh è stata generata automaticamente dal software una volta imposta la dimensione dell'elemento $d$ con il comando 'global element size'. Nessun altro controllo sulla forma e dimensione degli elementi è stato utilizzato.

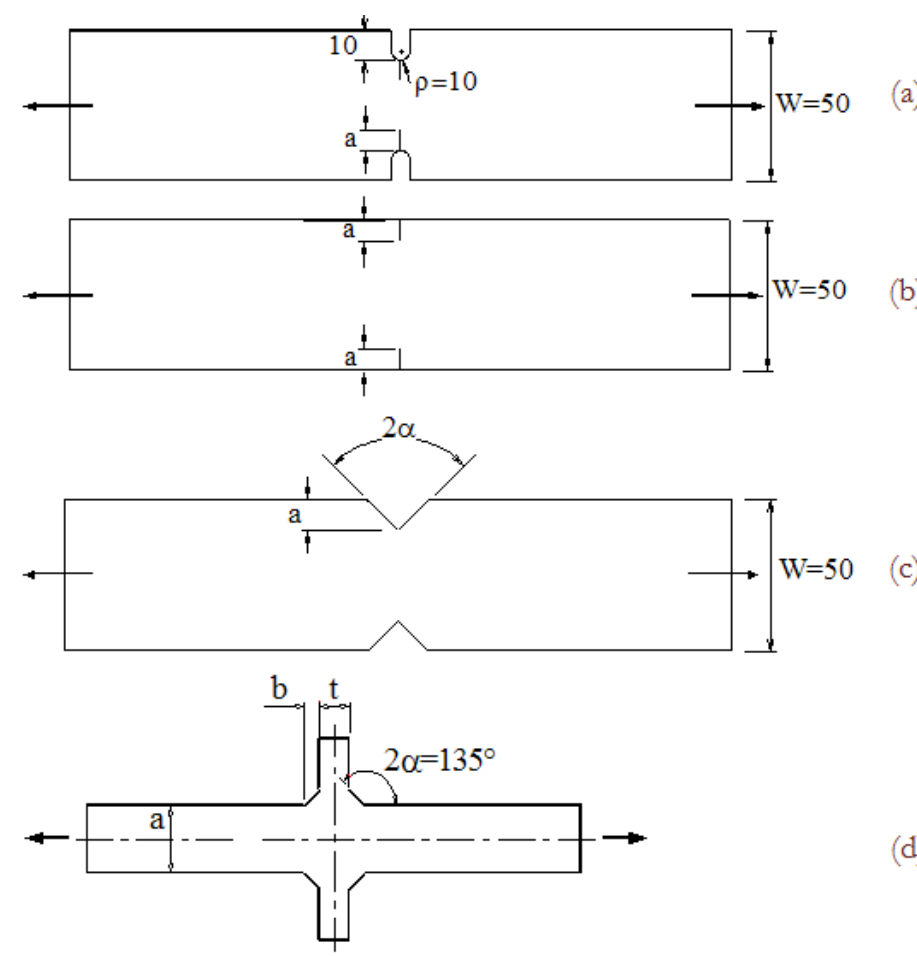

Figura 3: geometrie contenenti punti di singolarità tensionale utilizzate per validare il metodo della tensione di picco (dimensioni in $\mathrm{mm}$ ) [8]. 
I risultati sono riportati nella Fig. 4, che mostra, al variare del rapporto tra la dimensione di riferimento a del componente e la dimensione $\mathrm{d}$ dell'elemento, il parametro adimensionale $\mathrm{K}_{\mathrm{FE}}^{*}$ :

$$
\mathrm{K}_{\mathrm{FE}}^{*}=\frac{\mathrm{K}_{\mathrm{I}}^{\mathrm{V}}}{\sigma_{\text {peak }} \cdot \mathrm{d}^{1-\lambda_{1}}}
$$

calcolato per ogni geometria presa in considerazione. La Fig. 4 mostra che non appena a/d è maggiore o uguale a 3, il parametro $\mathrm{K}_{\mathrm{FE}}^{*}$ è pressoché costante e pari a 1.38, con risultati numerici raccolti in una ristretta banda definita dal +-3\% rispetto a tale valore. In [8] è fornita anche un'espressione analitica per calcolare il parametro $\mathrm{K}_{\mathrm{FE}}^{*}$. Scelta quindi una certa dimensione di elemento d da utilizzare nelle analisi agli elementi finiti, la Fig. 4 e l'Eq. (3), dove $\mathrm{K}_{\mathrm{FE}}^{*}=1.38$, mostrano che il rapporto $\mathrm{K}_{\mathrm{I}}^{\mathrm{V}} / \sigma_{\text {peak }}$ è costante e quindi la tensione di picco $\sigma_{\text {peak }}$ può sostituire l'N-SIF $\mathrm{K}_{\mathrm{I}}^{\mathrm{V}}$ nelle analisi di resistenza a fatica con notevole riduzione dei tempi e semplificazione delle procedure di calcolo. È necessario tener presente che il metodo della tensione di picco così come presentato è valido sotto le seguenti condizioni:

- Elementi lineari quadrilateri a quattro nodi (PLANE 42, della libreria del codice ANSYS 8.0);

- Pattern di elementi come quello riportato nella successiva Fig. 5;

- Intagli a $\mathrm{V}$ con angolo di apertura compreso tra $0^{\circ}$ e $135^{\circ}$.

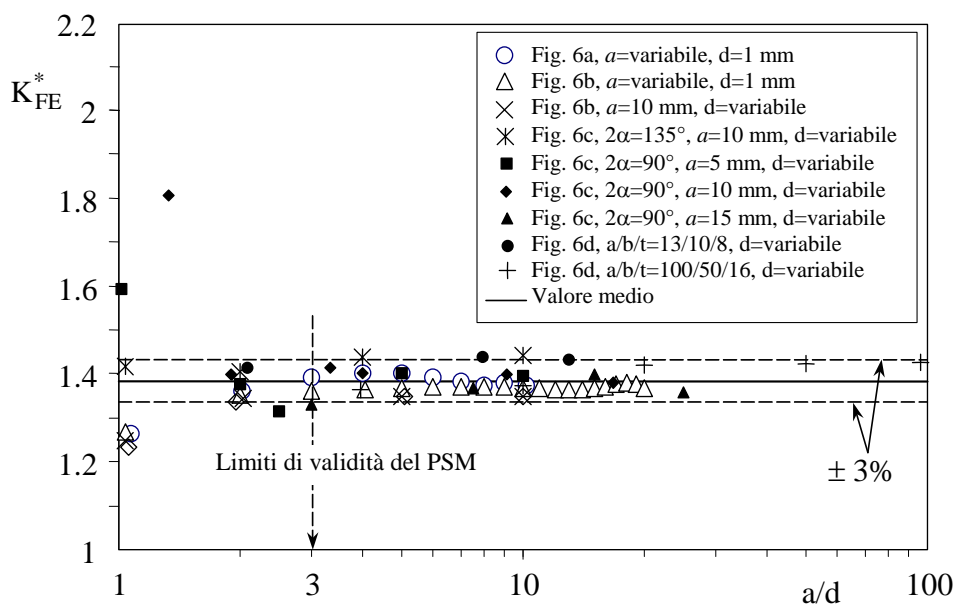

Figura 4: parametro adimensionale $\mathrm{K}_{\mathrm{FE}}^{*}$ ottenuto da 61 analisi agli elementi finiti delle geometrie riportate in Fig. 5. La linea continua rappresenta il valor medio pari a 1.38 [8].

\section{DEFINIZIONE DI CURVE DI RESISTENZA A FATICA DI PROGETTO PER GIUNTI SALDATI D'ANGOLO IN ACCIAIO E IN LEGA LEGGERA}

T

a possibilità di definire bande di resistenza a fatica unificate, al variare della geometria e delle dimensioni assolute, per giunti in acciaio e in lega leggera saldati d'angolo in termini di range di variazione di $\mathrm{K}_{\mathrm{I}}^{\mathrm{V}}, \Delta \mathrm{K}_{\mathrm{I}}^{\mathrm{V}}$, è già stata

verificata in passato $[1,3]$. Essendo valida l'Eq. (3) $\operatorname{con} \mathrm{K}_{\mathrm{FE}}^{*}=1.38$ e una volta scelta la dimensione di elemento utilizzata nell'analisi agli elementi finiti, è quindi possibile definire delle curve di progetto di resistenza a fatica in termini di range della tensione di picco $\Delta \sigma_{\text {peak. }}$ A tale scopo sono stati utilizzati i dati di Maddox [9], Gurney [10, 11], Kihl and Sarkani $[12,13]$ pubblicati negli articoli originali in termini di tensione nominale. I dettagli sulle geometrie, i materiali e le condizioni di carico sono riportati in [8]. In sintesi, i dati si riferiscono a giunti in acciaio da costruzione (con tensione di snervamento variabile tra 360 e $670 \mathrm{MPa}$ ) e in leghe leggere della serie 5000 e 6000 (con tensione di snervamento variabile fra 250 e $304 \mathrm{MPa}$ ) sollecitati a trazione o flessione con rapporto nominale di ciclo $\mathrm{R}$ (definito come rapporto fra la tensione nominale minima e quella massima applicata) prossimo a zero. Tutti i giunti hanno semplice configurazione a $\mathrm{T}$ o a croce con saldature d'angolo e sono stati sottoposti a prova senza alcun trattamento post-saldatura. I giunti in acciaio 
hanno spessore del piatto principale variabile fra 6 e $100 \mathrm{~mm}$ con rapporto tra gli spessori saldati compreso fra 0.03 e 8.8 , mentre quelli in lega leggera fra 3 e $24 \mathrm{~mm}$ con rapporto tra gli spessori saldati variabile fra 0.25 e 1.

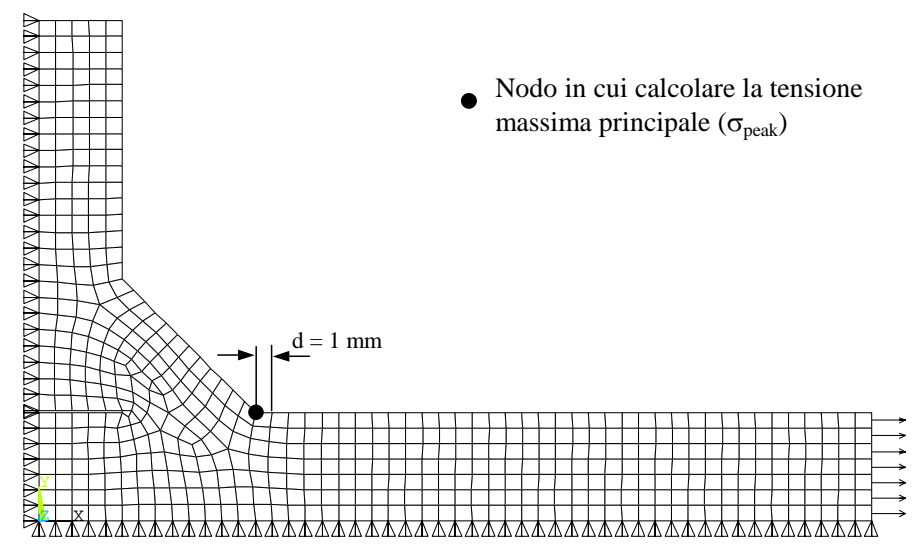

Figura 5: mesh free utilizzata per le analisi agli elementi finiti di giunti saldati d'angolo con elementi di dimensione pari a $1 \mathrm{~mm}$.

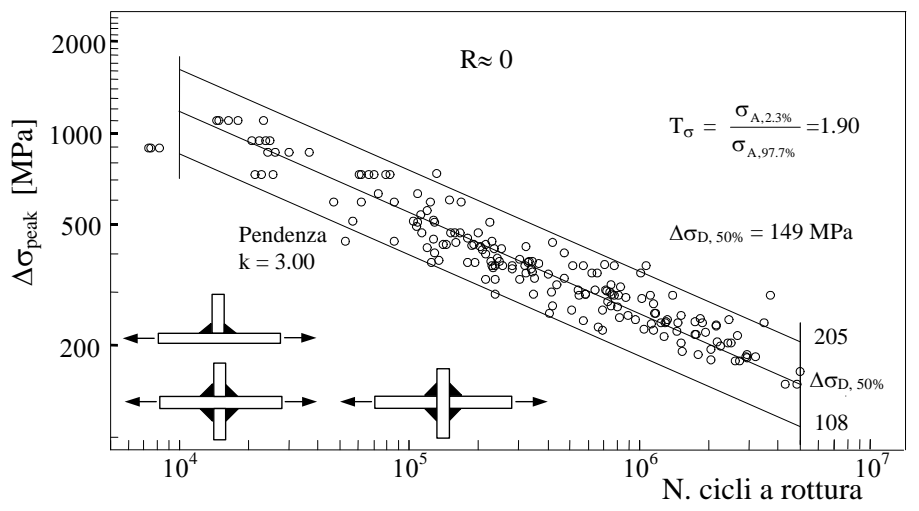

Figura 6: resistenza a fatica di giunti saldati d'angolo in acciaio da costruzione in termini di tensione di picco lineare elastica calcolata con la mesh riportata in Fig. 5. Banda di dispersione relativa al valor medio \pm due deviazioni standard [8].

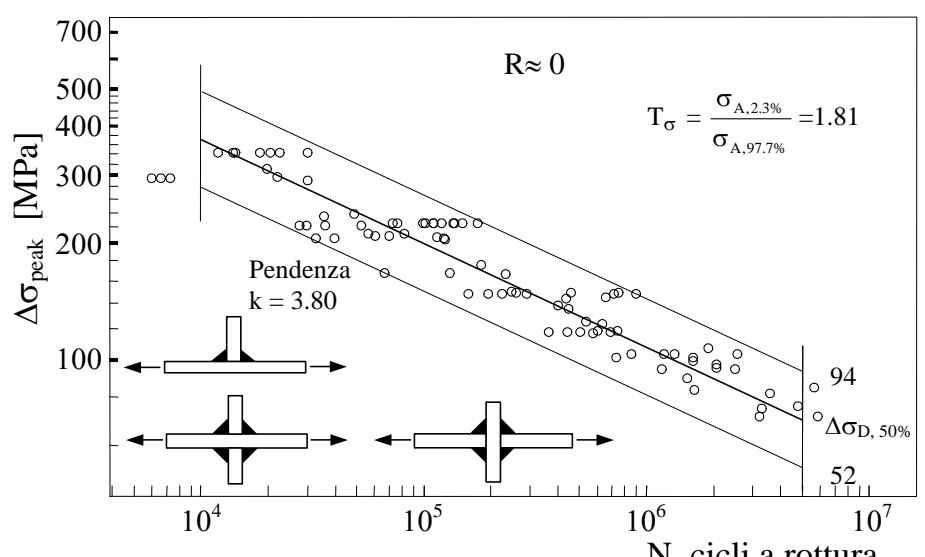

Figura 7: resistenza a fatica di giunti saldati d'angolo in lega leggera in termini di tensione di picco lineare elastica calcolata usando elementi di $1 \mathrm{~mm}$ di dimensione come riportato in Fig. 5. Banda di dispersione relativa al valor medio \pm due deviazioni standard [8].

Le analisi numeriche sono state fatte con il software Ansys usando mesh di tipo free con elementi di dimensione di $1 \mathrm{~mm}$ come riportato in Fig. 5 e imponendo una tensione nominale pari a $1 \mathrm{MPa}$. Calcolando la tensione massima principale $\left(\sigma_{\text {peak }}\right)$ nel punto di singolarità è stato possibile esprimere i risultati originali in termini di range di tensione di picco anziché 
range di tensione nominale. I risultati sono riportati in Fig. 6 e 7 per i giunti in acciaio e in lega leggera, rispettivamente. Le bande di dispersione riportate si riferiscono a probabilità di sopravvivenza del $97.7 \%$ e il parametro $T_{\sigma}$ che ne quantifica l'ampiezza risulta confrontabile con i valori trovati in termini di N-SIF [3]. Considerando ulteriormente i giunti in acciaio e calcolando il parametro $T_{\sigma}$ per probabilità di sopravvivenza del 10\% e $90 \%$ si trova 1.51 in ottimo accordo con il valore di 1.50 relativo alla banda unificata proposta da Haibach [14]. Pertanto è possibile concludere che le bande riportate nelle Fig. 6 e 7 si possono ritenere bande unificate per la progettazione a fatica di giunti saldati d'angolo in acciaio da costruzione e lega leggera, rispettivamente, senza trattamenti post-saldatura e per rotture innescate a piede cordone.

\section{APPLICAZIONE DEL METODO DELLA TENSIONE DI PICCO A GIUNTI COMPLESSI}

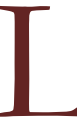

e curve di resistenza a fatica riportate nelle Fig. 6 e 7 sono state utilizzate per stimare la resistenza a fatica di giunti saldati d'angolo aventi geometria complessa presenti in letteratura.

Le Tab. 1 e 2 riportano le geometrie di giunto analizzate per acciai e leghe leggere, rispettivamente, mentre per i dettagli riguardo ai materiali e ai riferimenti ai lavori originali si rimanda a [15]. Le geometrie dei giunti sono del tipo con irrigidimento longitudinale, single-lap o tubolare come riportato nelle Tab. 1 e 2. Per quanto riguarda i giunti in acciaio, $i$ materiali impiegati sono acciai da costruzione con tensione di snervamento compresa fra 309 e $780 \mathrm{MPa}$, gli spessori variabili fra 3 e $12.5 \mathrm{~mm}$, mentre il rapporto di ciclo è compreso fra 0 e 0.7 . Per quanto riguarda i giunti in lega leggera, i materiali impiegati sono leghe della serie 5000 e 6000 aventi tensione di snervamento compresa fra 245 e $290 \mathrm{MPa}$, gli spessori sono variabili fra 3 e $12 \mathrm{~mm}$, mentre il rapporto di ciclo è compreso è prossimo a zero.

Le analisi agli elementi finiti dei giunti bidimensionali, come ad esempio i giunti single-lap, sono state condotte utilizzando la mesh riportata in Figura 5, mentre per calcolare la tensione di picco nel caso di giunti tubolari è stata utilizzata la procedura a due step dettagliata in $[15,16]$ e riassunta dalla Fig. 8.

Nel primo step di analisi è stato definito un modello ad elementi shell (modello principale). Nel secondo step di analisi si è immaginato di fare una sezione longitudinale del giunto reale in corrispondenza del punto di innesco della rottura a fatica del giunto con un piano che nel caso di Fig. 8 stacca sulla superficie del modello principale le due linee spesse. Si ottiene così il sottomodello riportato in Fig. 8 che riproduce lo spessore degli elementi saldati e il profilo a V a spigolo vivo del piede del cordone. I risultati dell'analisi sul modello principale, a cui sono applicate le condizioni al contorno, sono trasferiti al sottomodello imponendo una condizione di congruenza, ovvero gli spostamenti calcolati lungo le linee spesse del modello principale vengono imposti alle corrispondenti linee spesse del sottomodello. Questa operazione è effettuata automaticamente in ambiente Ansys utilizzando il comando 'shell-to-solid' submodeling.

Le Fig. 9 e 10 riportano il confronto fra i risultati sperimentali e le curve di progetto riportate nelle Fig. 6 e 7 , rispettivamente, per $\mathrm{i}$ giunti in acciaio e in lega leggera. Dal confronto si nota la validità delle curve di progetto, fatta eccezione per i giunti di più piccolo spessore, che meritano ulteriori approfondimenti [15]. Nelle strutture reali i percorsi di propagazione possono essere multipli oppure particolarmente lunghi, in modo tale da rendere significativa la quota parte di vita a fatica spesa per la propagazione di cricche al di fuori del piccolo volume di materiale in cui lo stato di tensione è controllato dagli N-SIFs. In questi casi le curve di progetto riportate nelle Figure 6 e 7 vanno utilizzate per stimare la vita fatica a innesco, mentre per la fase di propagazione è valido il classico approccio basato sulla Meccanica della Frattura Lineare Elastica.

Si ritiene infine che utilizzando il metodo della tensione di picco unitamente alla procedura di calcolo descritta, l'approccio N-SIF per la progettazione a fatica dei giunti saldati possa essere implementato in un contesto industriale.

\section{CONCLUSIONI}

I 1 metodo della tensione di picco (PSM) è stato applicato all'analisi della resistenza a fatica di giunti saldati d'angolo in acciaio e in lega leggera senza trattamenti post-saldatura con rottura al piede del cordone di saldatura. Il metodo proposto è un'applicazione ingegneristica dell'approccio locale basato sugli N-SIF, che assimila il profilo geometrico del piede del cordone di saldatura ad un intaglio a $\mathrm{V}$ a spigolo vivo con angolo di apertura tipicamente pari a $135^{\circ}$. Il metodo della tensione di picco utilizza come tensione di progetto la tensione di picco singolare calcolata al piede cordone con un'analisi agli elementi in cui la dimensione degli elementi sia prefissata, pari a $1 \mathrm{~mm}$ in questo lavoro. La tecnica di analisi è presentata nelle Figure 5 e 8 per giunti aventi geometria riconducibile a modelli bidimensionali e tridimensionali, rispettivamente. Le curve di resistenza a fatica da utilizzare per le verifiche sono riportate nelle Figure 6 e 7 , rispettivamente, per giunti in acciaio e in lega leggera. 


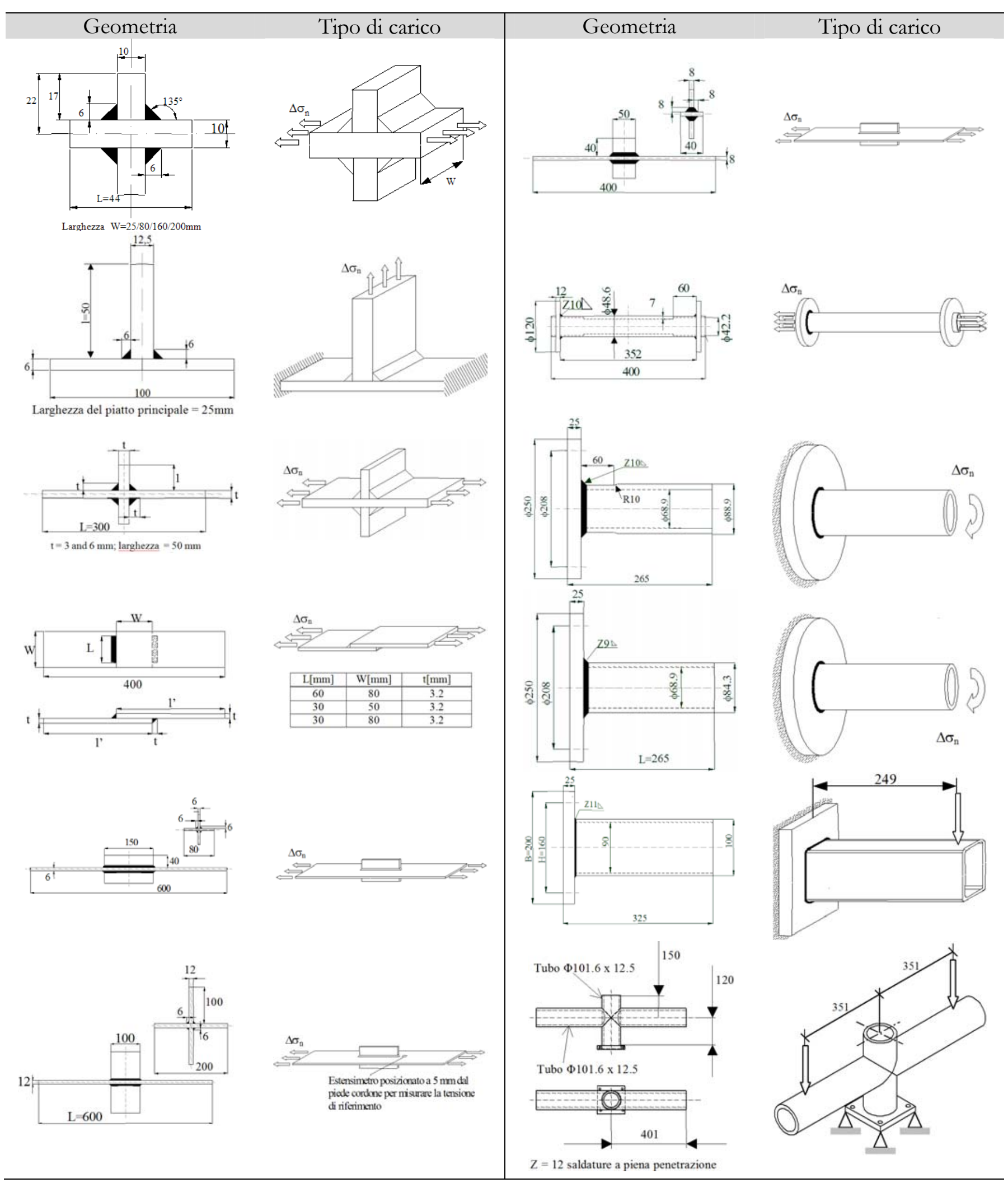

Tabella 1: Geometria e condizioni di carico per i giunti saldati d'angolo in acciaio considerati $\left(\Delta \sigma_{\mathrm{n}}\right.$ : range della tensione nominale considerata negli articoli originali; dimensioni in $\left.\mathrm{mm}\right)$ [15]. 


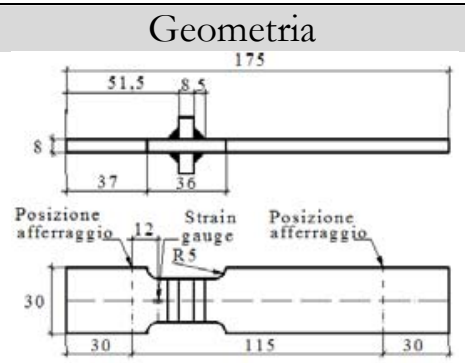

Tipo di carico
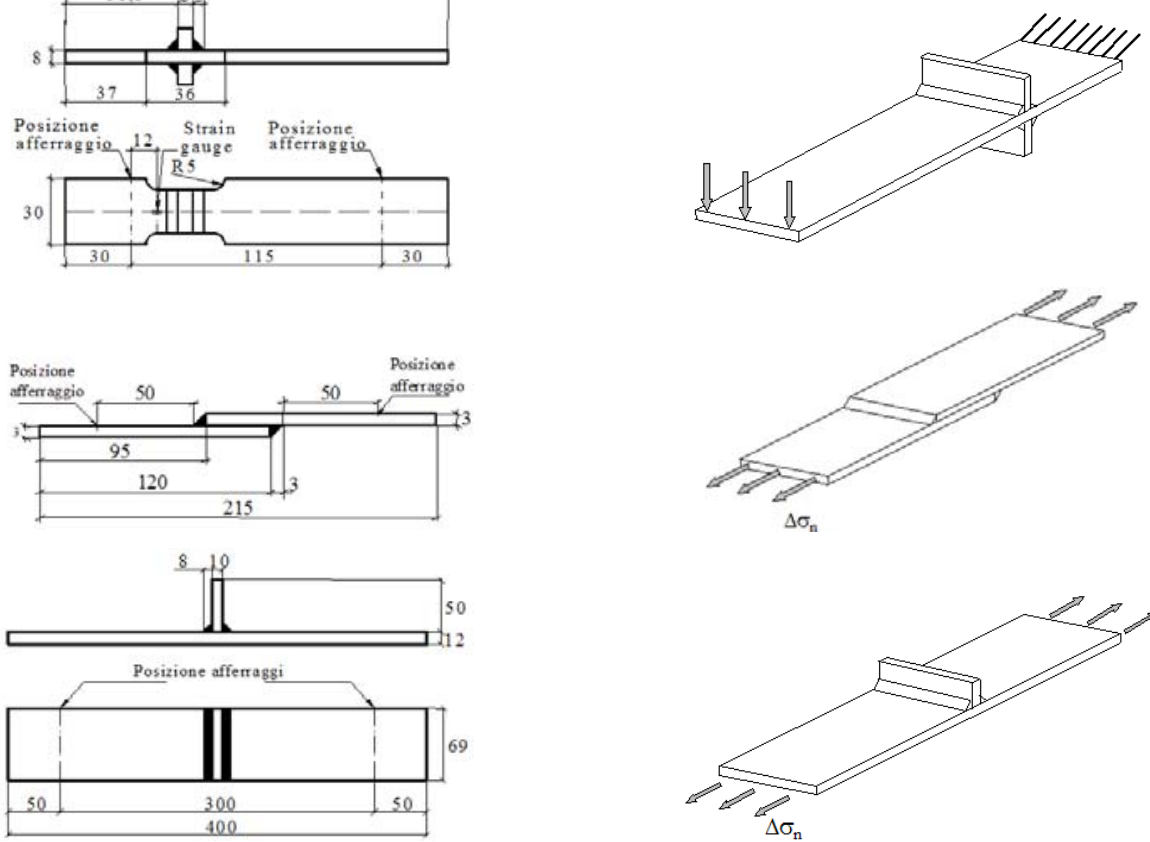

$\Delta \sigma_{\mathrm{r}}$
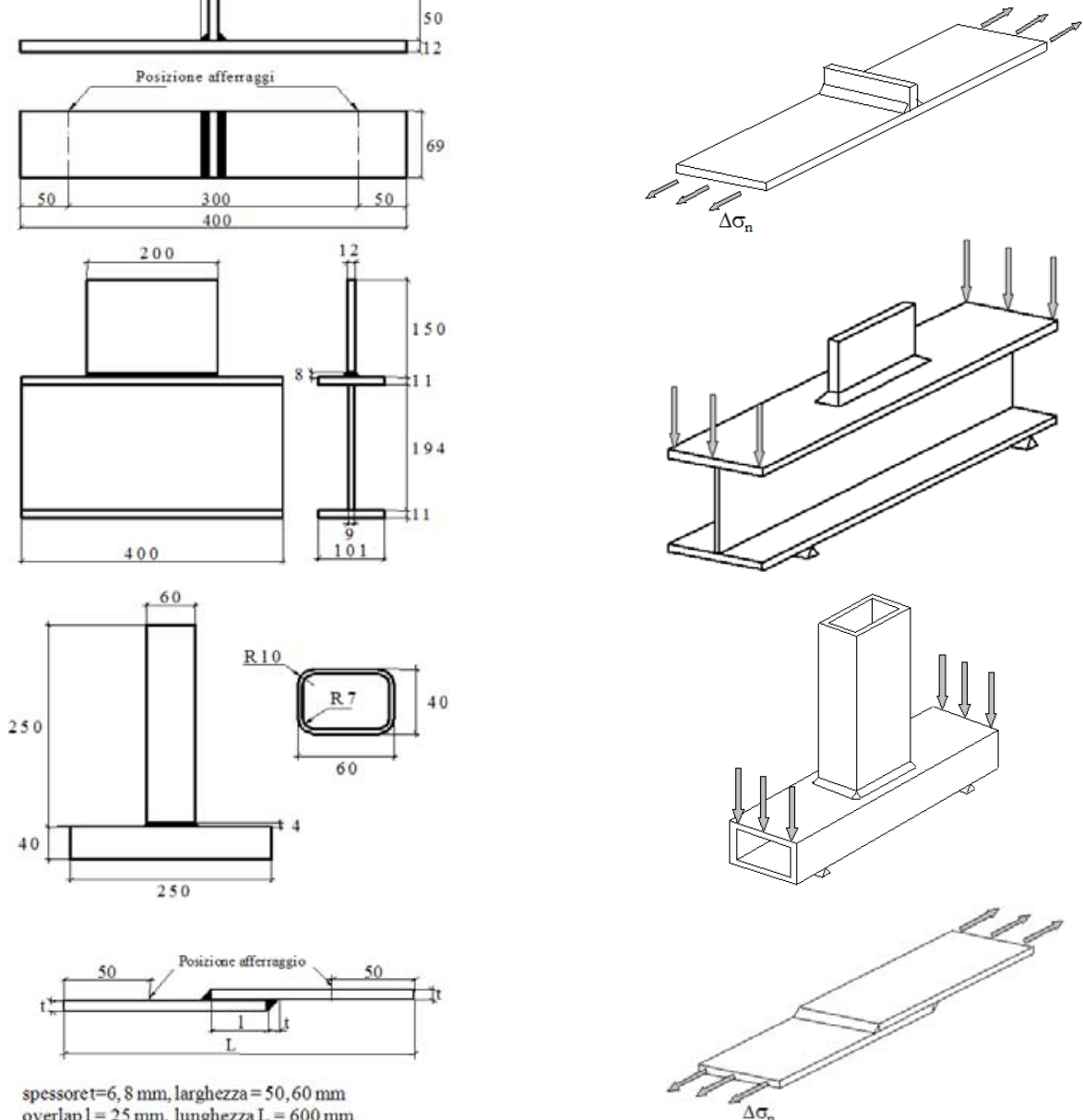

spessoret $=6,8 \mathrm{~mm}$, larghezza $=50,60 \mathrm{~mm}$ overlap $1=25 \mathrm{~mm}$, lunghezza $\mathrm{L}=600 \mathrm{~mm}$
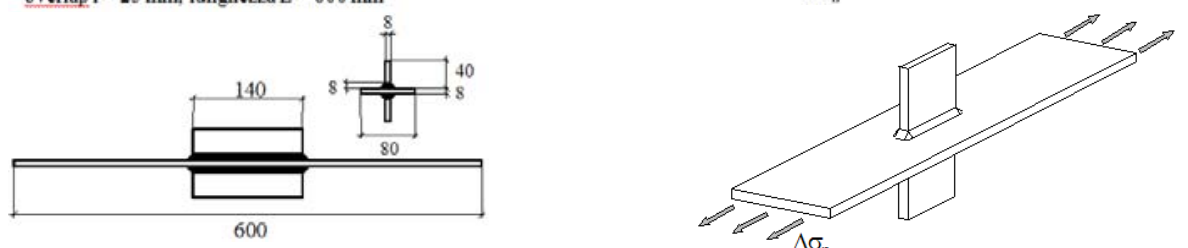

Tabella 2: Geometria e condizioni di carico per i giunti saldati d'angolo in lega leggera considerati $\left(\Delta \sigma_{\mathrm{n}}\right.$ : range della tensione nominale considerata negli articoli originali; dimensioni in $\left.\mathrm{mm}\right)$ [15]. 


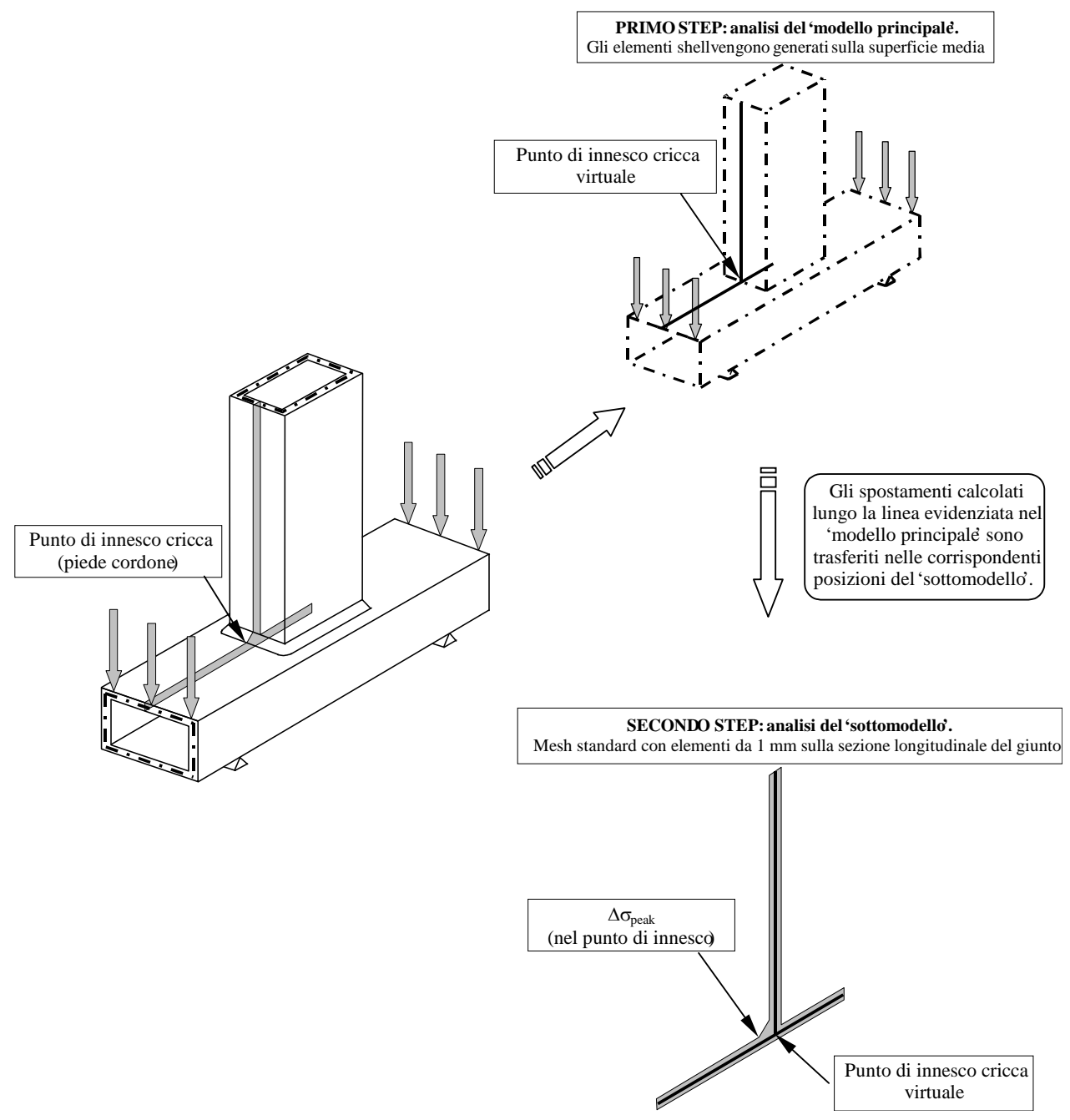

Figura 8: Tecnica di analisi agli elementi finiti di giunti tridimensionali per calcolare la tensione di picco $\left(\sigma_{\text {peak }}\right)$ a piede cordone [15].

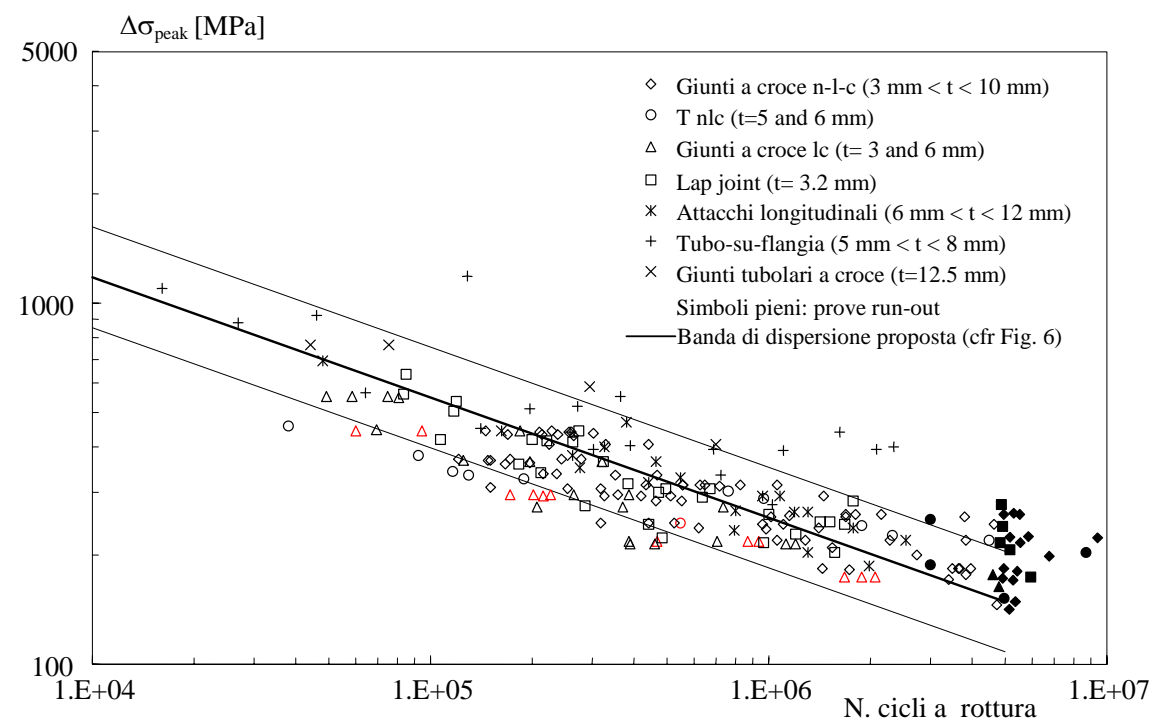

Figura 9: Confronto fra la banda di dispersione proposta in Fig. 6 e i risultati sperimentali in termini di range di tensione di picco per giunti saldati d'angolo in acciaio (n-l-c: cordoni non portanti; lc: cordoni portanti) [15]. 


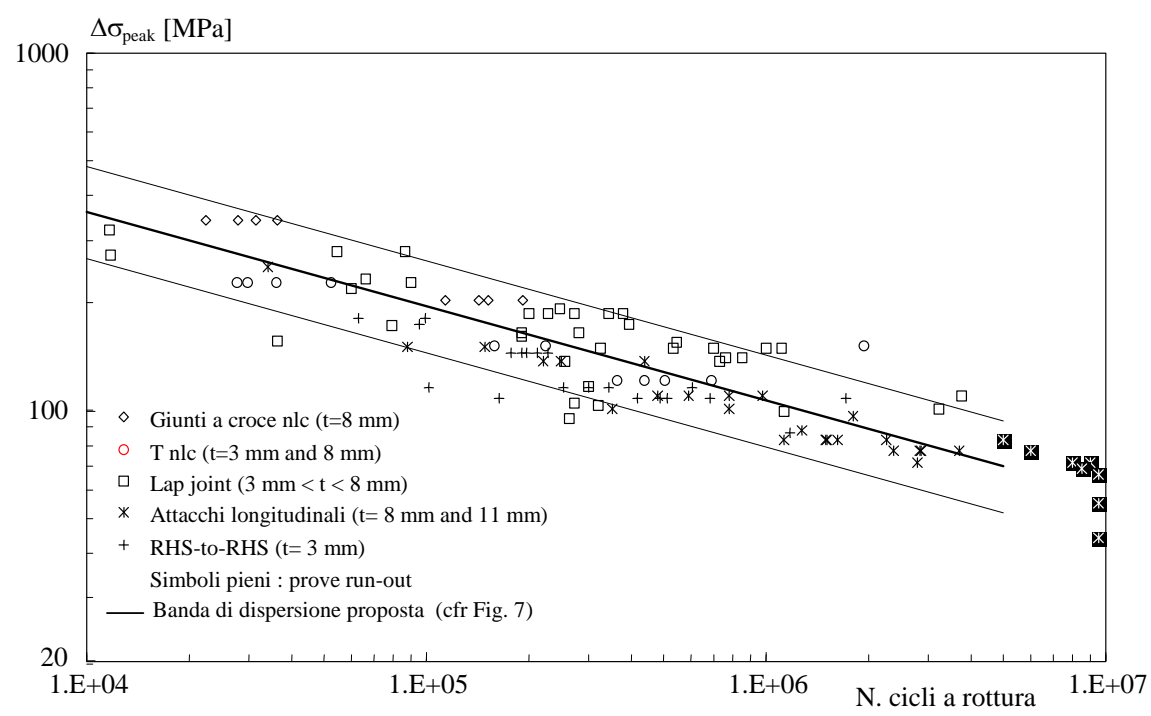

Figura 10: Confronto fra la banda di dispersione proposta in Fig. 6 e i risultati sperimentali in termini di range di tensione di picco per giunti saldati d'angolo in lega leggera (n-l-c: cordoni non portanti; lc: cordoni portanti; RHS-to-RHS: giunto realizzato con elementi tubolari a sezione rettangolare) [15].

\section{BIBLIOGRAFIA}

[1] P. Lazzarin, R. Tovo, Fatigue Fract. Engng. Mater. Struct., 21 (1998)1089.

[2] B. Atzori, G. Meneghetti, Int. J. Fatigue, 23(8) (2001) 713.

[3] P. Lazzarin, P. Livieri, Int. J. Fatigue, 23 (2001) 225.

[4] A. Seto, Y. Yoshida, A. Galtier, Fatigue Fract. Engng. Mater. Struct., 27 (2004) 1147.

[5] M. L. Williams, J. Appl. Mech., 19 (1952) 526.

[6] R. Gross, A. Mendelson, Int. J. Fract. Mech., 8 (1972) 267.

[7] H. Nisitani, T. Teranishi, Engng. Fract. Mech., 71 (2004) 579.

[8] G. Meneghetti, P. Lazzarin, Fatigue Fract. Engng. Mater. Struct., 30 (2007) 95.

[9] S. J. Maddox, The effect of plate thickness on the fatigue strength of fillet welded joints, Abington Publishing, Abington, Cambridge (1987).

[10] T. R. Gurney, The fatigue strength of transverse fillet welded joints, Abington Publishing, Abington, Cambridge (1991).

[11] T. R. Gurney, Fatigue of thin walled joints under complex loading, Abington Publishing, Abington, Cambridge (1997).

[12] D. P. Kihl, S. Sarkani, Int. J. Fatigue, 19 (1997) S311.

[13] D. P. Kihl, S. Sarkani, Probab. Eng. Mech. , 14 (1999) 97.

[14] E. Haibach Service fatigue-strength - methods and data for structural analysis, Dusseldorf, VDI (1989).

[15] G. Meneghetti, Fatigue Fract. Engng. Mater. Struct., 31 (5) (2008) 346.

[16] G. Meneghetti, F. Righetto, Atti del XXXV Convegno Nazionale AIAS, Ancona (2006). 